KIAS-P010015

\title{
Dark matter and a new gauge boson through kinetic mixing
}

\author{
Eung Jin Chun and Jong-Chul Park \\ Korea Institute for Advanced Study \\ Heogiro 87, Dongdaemun-gu \\ Seoul 130-722, Korea \\ Emails: ejchun@kias.re.kr, jcpark@kias.re.kr \\ Stefano Scopel \\ Department of Physics, Sogang University \\ 1 Sinsu-dong, Mapo-gu \\ Seoul 121-742, Korea \\ Email: scopel@sogang.ac.kn
}

\begin{abstract}
We consider a hidden sector model of dark matter which is charged under a hidden $U(1)_{X}$ gauge symmetry. Kinetic mixing of $U(1)_{X}$ with the Standard Model hypercharge $U(1)_{Y}$ is allowed to provide communication between the hidden sector and the Standard Model sector. We present various limits on the kinetic mixing parameter and the hidden gauge coupling constant coming from various low energy observables, electroweak precision tests, and the right thermal relic density of the dark matter. Saturating these constraints, we show that the spin-independent elastic cross section of the dark matter off nucleons is mostly below the current experimental limits, but within the future sensitivity. Finally, we analyze the prospect of observing the hidden gauge boson through its dimuon decay channel at hadron colliders.
\end{abstract}

KEYWORDS: Hidden gauge symmetry, Kinetic mixing, Thermal dark matter. 


\section{Contents}

1. Introduction 1

2. Hidden $U(1)_{X}$ model and gauge interactions 2

3. Low-energy and electroweak constraints 5

$3.1 g_{\mu}-2$

3.2 Parity-violation effect in atomic physics 6

$3.3 \rho$ parameter 6

3.4 EWPT 9

4. Thermal relic abundance of dark matter 10

5. Direct detection of dark matter 12

6. Tevatron and $\mathrm{LHC}$ probes of $\mathrm{U}(1)_{\mathrm{X}}$

7. Conclusions 17

A. Interaction couplings 20

\section{Introduction}

One of the popular scenarios for a TeV-scale physics beyond the Standard Model is postulating an additional gauge interaction other than the Standard Model one $S U(3)_{c} \times S U(2)_{L} \times U(1)_{Y}$. A classic example is an extra $U(1)$ interaction that arises from a grand unification theory [1]. Such a possibility has been well studied as it might be discovered in the early stage of the LHC experiment. Another interesting possibility for an extended gauge sector is to assume a $U(1)_{X}$ interaction in the hidden sector, in the sense that Standard Model particles are neutral under $U(1)_{X}$. However, the hidden sector and the Standard Model sector can couple to each other through the kinetic mixing of $U(1)_{X}$ and $U(1)_{Y}$ [2]. Since the kinetic mixing term is gauge-invariant, it can be present at the tree-level. In the hidden $U(1)_{X}$ model, one can introduce a massive Dirac fermion charged under $U(1)_{X}$ which is a dark matter (DM) candidate. This kind of scenario has been used to implement $\mathrm{MeV}$ DM [3], a sub-GeV $X$ boson for the Sommerfeld enhancement employing kinetic and 
Higgs mixing [4], light DM with Sommerfeld enhancement in the NMSSM with gauge mediation [5], a TeV scale hidden sector through Higgs mixing [6], and a $10 \mathrm{GeV}$ DM through kinetic mixing [7]. Let us also remark that Stuekelberg $Z^{\prime}$ models have similar features [8, 9, 10].

In this paper, we examine various phenomenological implications of the kinetic mixing of $U(1)_{X}$ and of a DM candidate which has a preferable mass scale of $0.1-1$ $\mathrm{TeV}$. In this model, the hidden dark matter physics is described by four parameters: the kinetic mixing angle $\epsilon$, the dark matter mass $m_{\psi}$, the $X$ boson mass $m_{X}$, and the $X$ gauge coupling $g_{X}$. These parameters are constrained by various low-energy and electroweak observables, Tevatron II results, and the thermal relic density of dark matter. After considering these constraints, we will look for perspectives for the direct detection of dark matter and the LHC discovery of the $X$ boson.

This paper is organized as follows. In Section 2, we set up the hidden sector dark matter model and present interaction vertices relevant for further discussions. In Section 3, we work out various constraints on the kinetic mixing parameter $\epsilon$ from low-energy observables [11, 12] and electroweak precision tests [13, 14] used in the phenomenological discussion of the ensuing Sections. In Section 1 , the DM annihilation rate is calculated and normalized to the observed DM relic density. In particular, by using this procedure we fix a combination of $\epsilon$ and $g_{X}\left(\simeq \epsilon g_{X}\right.$ in the limit $\epsilon \ll 1$ ) as a function of the two masses $m_{\psi}$ and $m_{X}$. In Section 5, the spin-independent DM-nucleon cross section is calculated and compared to the recent CDMS II [15] and XENON100 [16] results. In Section 6, we analyze the Tevatron II limit on the $X$ boson production and dimuon decay and the LHC perspective for detection of the same quantity, which depends on the branching ratio of the $\mathrm{X}$ boson decay to the dark matter particle. Finally, we conclude in Section 0 .

\section{Hidden $U(1)_{X}$ model and gauge interactions}

We consider a hidden sector containing a gauge symmetry $U(1)_{X}$ and a Dirac fermion dark matter candidate at the TeV scale, which couples to the Standard Model sector through kinetic mixing. The full Lagrangian including kinetic mixing is:

$$
\mathcal{L}=\mathcal{L}_{S M}-\frac{1}{2} \sin \epsilon \hat{B}_{\mu \nu} \hat{X}^{\mu \nu}-\frac{1}{4} \hat{X}^{\mu \nu} \hat{X}_{\mu \nu}-g_{X} \hat{X}^{\mu} \bar{\psi} \gamma_{\mu} \psi+\frac{1}{2} m_{\hat{X}^{2}}^{2} \hat{X}^{2}+m_{\psi} \bar{\psi} \psi
$$

where the $U(1)_{X}$ is assumed to be broken spontaneously leading to the gauge boson

mass $m_{\hat{X}}$. In the Standard Model sector, the $\hat{Z}$ gauge boson has the mass $m_{\hat{Z}}$ and the gauge couplings are denoted by $\hat{g}=\hat{e} / s_{\hat{W}}$ and $\hat{g}^{\prime}=\hat{e} / c_{\hat{W}}$. Diagonalizing away the kinetic mixing term and mass mixing terms is made by the following transformation 
[17]:

$$
\begin{aligned}
\hat{B} & =c_{\hat{W}} A-\left(t_{\epsilon} s_{\xi}+s_{\hat{W}} c_{\xi}\right) Z+\left(s_{\hat{W}} s_{\xi}-t_{\epsilon} c_{\xi}\right) X \\
\hat{W}_{3} & =s_{\hat{W}} A+c_{\hat{W}} c_{\xi} Z-c_{\hat{W}} s_{\xi} X \\
\hat{X} & =\frac{s_{\xi}}{c_{\epsilon}} Z+\frac{c_{\xi}}{c_{\epsilon}} X
\end{aligned}
$$

where the angle $\xi$ is determined by:

$$
\tan 2 \xi=-\frac{m_{\hat{Z}}^{2} s_{\hat{W}} \sin 2 \epsilon}{m_{\hat{X}}^{2}-m_{\hat{Z}}^{2}\left(c_{\epsilon}^{2}-s_{\epsilon}^{2} s_{\hat{W}}^{2}\right)} .
$$

Then, the $X$ and $Z$ gauge bosons get the redefined masses:

$$
\begin{aligned}
& m_{Z}^{2}=m_{\hat{Z}}^{2}\left(1+s_{\hat{W}} t_{\xi} t_{\epsilon}\right), \\
& m_{X}^{2}=\frac{m_{\hat{X}}^{2}}{c_{\epsilon}^{2}\left(1+s_{\hat{W}} t_{\xi} t_{\epsilon}\right)} .
\end{aligned}
$$

Moreover, from Eqs. (2.3) - (2.5) one can find $t_{\xi}$ as a function of $r_{X} \equiv m_{X}^{2} / m_{Z}^{2}$ :

$$
\begin{aligned}
t_{\xi} & =-\frac{1}{s_{\hat{W}} t_{\epsilon}}, \\
t_{\xi} & =\frac{1-r_{X} \pm \sqrt{\left(1-r_{X}\right)^{2}-4 s_{\hat{W}}^{2} t_{\epsilon}^{2} r_{X}}}{2 s_{\hat{W}} t_{\epsilon} r_{X}} .
\end{aligned}
$$

The solution given by Eq.(2.6) is unphysical since it corresponds to $m_{Z}=0$ and $m_{X}=\infty$. In addition, the other two solutions (2.7) are physical only in the range $r_{X} \leq 1+2 s_{\hat{W}}^{2} t_{\epsilon}^{2}-2 \sqrt{s_{\hat{W}}^{2} t_{\epsilon}^{2}\left(1+s_{\hat{W}}^{2} t_{\epsilon}^{2}\right)}$ or $r_{X} \geq 1+2 s_{\hat{W}}^{2} t_{\epsilon}^{2}+2 \sqrt{s_{\hat{W}}^{2} t_{\epsilon}^{2}\left(1+s_{\hat{W}}^{2} t_{\epsilon}^{2}\right)}$, where $\left(1-r_{X}\right)^{2}-4 s_{\hat{W}}^{2} t_{\epsilon}^{2} r_{X}^{2}>0$. In the following analysis we exclude the unphysical region, which is limited to a small interval around $m_{X}=m_{Z}$. As we will see shortly, the weak mixing angle $s_{\hat{W}}$ is very close to the physical angle $s_{W}$ due to the strong $\rho$ parameter limit.

Let us now list all the interaction vertices relevant for our analysis. The $W, Z$, and $X$ gauge bosons have the following fermion couplings:

$$
\begin{aligned}
& \mathcal{L}_{W}=-\frac{e}{\sqrt{2} s_{\hat{W}}} W_{\mu}^{+}\left\{\bar{\nu} \gamma^{\mu} P_{L} e+\bar{u} \gamma^{\mu} P_{L} d\right\}+c . c . \\
& \mathcal{L}_{Z}=-\frac{e}{c_{\hat{W}} s_{\hat{W}}} c_{\xi} Z_{\mu} \bar{f} \gamma^{\mu}\left\{P_{L}\left[T_{3}\left(1+s_{\hat{W}} t_{\epsilon} t_{\xi}\right)-Q\left(s_{\hat{W}}^{2}+s_{\hat{W}} t_{\epsilon} t_{\xi}\right)\right]\right. \\
&\left.-P_{R}\left[Q\left(s_{\hat{W}}^{2}+s_{\hat{W}} t_{\epsilon} t_{\xi}\right)\right]\right\} f-g_{X} \frac{s_{\xi}}{c_{\epsilon}} Z_{\mu} \bar{\psi} \gamma^{\mu} \psi, \\
& \mathcal{L}_{X}=-\frac{e}{c_{\hat{W}} s_{\hat{W}}} c_{\xi} X_{\mu} \bar{f} \gamma^{\mu}\left\{P_{L}\left[T_{3}\left(s_{\hat{W}} t_{\epsilon}-t_{\xi}\right)+Q\left(s_{\hat{W}}^{2} t_{\xi}-s_{\hat{W}} t_{\epsilon}\right)\right]\right. \\
&\left.+P_{R}\left[Q\left(s_{\hat{W}}^{2} t_{\xi}-s_{\hat{W}} t_{\epsilon}\right)\right]\right\} f-g_{X} \frac{c_{\xi}}{c_{\epsilon}} X_{\mu} \bar{\psi} \gamma^{\mu} \psi .
\end{aligned}
$$


The couplings of the $Z$ and $X$ gauge bosons to the $W$ gauge bosons are given by:

$$
\mathcal{L}_{V W W}=\frac{e}{t_{\hat{W}}} c_{\xi}\left[\left[Z W^{+} W^{-}\right]\right]-\frac{e}{t_{\hat{W}}} s_{\xi}\left[\left[X W^{+} W^{-}\right]\right],
$$

where $\left[\left[V W^{+} W^{-}\right]\right] \equiv i\left[\left(\partial_{\mu} W_{\nu}^{+}-\partial_{\nu} W_{\mu}^{+}\right) W^{\mu-} V^{\nu}-\left(\partial_{\mu} W_{\nu}^{-}-\partial_{\nu} W_{\mu}^{-}\right) W^{\mu+} V^{\nu}+(1 / 2)\left(\partial_{\mu} V_{\nu}-\right.\right.$ $\left.\left.\partial_{\nu} V_{\mu}\right)\left(W^{\mu+} W^{\nu-}-W^{\mu-} W^{\nu+}\right)\right]$. The couplings of the Higgs scalar $h$ to the $Z$ and $X$ gauge bosons are:

$$
\begin{aligned}
\mathcal{L}_{h V V}=\frac{m_{\hat{Z}}^{2}}{v} c_{\xi}^{2} h & {\left[\left(1+s_{\hat{W}} t_{\xi} t_{\epsilon}\right)^{2} Z_{\mu} Z^{\mu}+t_{\xi}^{2}\left(1-s_{\hat{W}} \frac{t_{\epsilon}}{t_{\xi}}\right)^{2} X_{\mu} X^{\mu}\right.} \\
& \left.+2 t_{\xi}\left(-1+2 s_{\hat{W}} \frac{t_{\epsilon}}{t_{\xi}}+s_{\hat{W}}^{2} t_{\epsilon}^{2}\right) X_{\mu} Z^{\mu}\right] .
\end{aligned}
$$

Summarizing the interaction vertices in Eqs. (2.8) - (2.12), let us define the various couplings, $g$ 's, as follows:

$$
\begin{aligned}
\mathcal{L} & =W_{\mu}^{+} g_{f}^{W}\left[\bar{\nu} \gamma^{\mu} P_{L} e+\bar{u} \gamma^{\mu} P_{L} d\right]+c . c . \\
& +Z_{\mu}\left[g_{f L}^{Z} \bar{f} \gamma^{\mu} P_{L} f+g_{f R}^{Z} \bar{f} \gamma^{\mu} P_{R} f+g_{\psi}^{Z} \bar{\psi} \gamma^{\mu} \psi\right]+g_{W}^{Z}\left[\left[Z W^{+} W^{-}\right]\right] \\
& +X_{\mu}\left[g_{f L}^{X} \bar{f} \gamma^{\mu} P_{L} f+g_{f R}^{X} \bar{f} \gamma^{\mu} P_{R} f+g_{\psi}^{X} \bar{\psi} \gamma^{\mu} \psi\right]++g_{W}^{X}\left[\left[X W^{+} W^{-}\right]\right] \\
& +h\left[g_{Z Z}^{h} Z_{\mu} Z^{\mu}+g_{X X}^{h} X_{\mu} X^{\mu}+g_{X Z}^{h} X_{\mu} Z^{\mu}\right]
\end{aligned}
$$

Unlike the $Z$ and $X$ gauge bosons, the mass of the $W$ gauge boson is not modified by the above transformation (2.2):

$$
m_{W}^{2}=m_{\hat{W}}^{2}=m_{\hat{Z}}^{2} c_{\hat{W}}^{2} .
$$

Then, the $\rho$ parameter is given by:

$$
\rho \equiv \frac{m_{W}^{2}}{m_{Z}^{2} c_{W}^{2}}=\frac{c_{\hat{W}}^{2}}{\left(1+s_{\hat{W}} t_{\xi} t_{\epsilon}\right) c_{W}^{2}} .
$$

Consequently, the current bound on the $\rho$ parameter, $\rho-1=4_{-4}^{+8} \times 10^{-4}$ [18], provides a constraint on the parameter $\epsilon$ as a function of the gauge boson masses. Let us note that the photon coupling does not change $(\hat{e}=e)$ and the identity $m_{Z}^{2} /\left(g^{2}+g^{\prime 2}\right)=m_{\hat{Z}}^{2} /\left(\hat{g}^{2}+\hat{g}^{\prime 2}\right)$ [17 leads to the relation between the original and redefined weak mixing angles:

$$
c_{W}^{2} s_{W}^{2}=\frac{c_{\hat{W}}^{2} s_{\hat{W}}^{2}}{1+s_{\hat{W}} t_{\xi} t_{\epsilon}} .
$$

Therefore, the $\rho$ parameter can be recast as

$$
\rho=\frac{s_{W}^{2}}{s_{\hat{W}}^{2}} .
$$


Defining $\hat{\delta} \equiv \rho-1=s_{W}^{2} / s_{\hat{W}}^{2}-1$ and expanding at the leading order in $\hat{\delta}$, we find:

$$
\omega \equiv s_{W} t_{\xi} t_{\epsilon} \simeq-\left(1-t_{W}^{2}\right) \hat{\delta}
$$

In the redefined physical basis, the above couplings can be rewritten in the first order of $\omega$ (or $\hat{\delta}$ ) as follows:

$$
\begin{aligned}
\mathcal{L}_{W}= & -\frac{e}{\sqrt{2} s_{W}}\left(1-\frac{\omega}{2\left(1-t_{W}^{2}\right)}\right) W_{\mu}^{+}\left\{\bar{\nu} \gamma^{\mu} P_{L} e+\bar{u} \gamma^{\mu} P_{L} d\right\}+c . c . \\
\mathcal{L}_{Z}= & -\frac{e}{c_{W} s_{W}} c_{\xi} Z_{\mu} \bar{f} \gamma^{\mu}\left\{P_{L} T_{3}\left[1+\frac{\omega}{2}\right]-Q\left[s_{W}^{2}+\omega\left(\frac{2-t_{W}^{2}}{2\left(1-t_{W}^{2}\right)}\right)\right]\right\} f \\
& -g_{X} \frac{s_{\xi}}{c_{\epsilon}} Z_{\mu} \bar{\psi} \gamma^{\mu} \psi \\
\mathcal{L}_{X}= & -\frac{e}{c_{W} s_{W}} c_{\xi} X_{\mu} \bar{f} \gamma^{\mu}\left\{P_{L} T_{3}\left[s_{W} t_{\epsilon}-t_{\xi}+\frac{1}{2} \omega\left(t_{\xi}+\frac{s_{W} t_{W}^{2} t_{\epsilon}}{1-t_{W}^{2}}\right)\right]\right. \\
& \left.\left.-g_{X} \frac{c_{\xi}}{c_{\epsilon}} X_{\mu} \bar{\psi} \gamma^{\mu} \psi, \quad+s_{W}^{2} t_{\xi}-s_{W} t_{\epsilon}+\frac{1}{2} t_{W}^{2} \omega\left(\frac{t_{\xi}-s_{W} t_{\epsilon}}{1-t_{W}^{2}}\right)\right]\right\} f \\
\mathcal{L}_{V W W}= & \frac{e}{t_{W}}\left(1-\frac{\omega}{2\left(c_{W}^{2}-s_{W}^{2}\right)}\right)\left\{c_{\xi}\left[\left[Z W^{+} W^{-}\right]\right]-s_{\xi}\left[\left[X W^{+} W^{-}\right]\right]\right\} \\
\mathcal{L}_{h V V}= & \frac{m_{Z}^{2}}{v} c_{\xi}^{2} h\left\{[1+\omega] Z_{\mu} Z^{\mu}+\left[t_{\xi}^{2}+s_{W}^{2} t_{\epsilon}^{2}-\omega\left(2+t_{\xi}^{2}-\frac{s_{W}^{2} t_{W}^{2} t_{\epsilon}^{2}}{1-t_{W}^{2}}\right)\right] X_{\mu} X^{\mu}\right. \\
& \left.+2\left[2 s_{W} t_{\epsilon}-t_{\xi}+\omega\left(t_{\xi}+\frac{s_{W} t_{W}^{2} t_{\epsilon}}{1-t_{W}^{2}}\right)\right] X_{\mu} Z^{\mu}\right\}
\end{aligned}
$$

From these expressions, we can read off the couplings defined in Eq. (2.13). The explicit expressions of the redefined interaction couplings are given in Appendix A.

\section{Low-energy and electroweak constraints}

The dark matter model with a hidden $U(1)_{X}$ sector introduced in the previous Section has four free parameters: $\epsilon, g_{X}, m_{X}$, and $m_{\psi}$. In this Section, we show how sizable the $U(1)_{X}$ contributions are for the muon $g-2$, atomic parity-violation, $\rho$ parameter and Electro-Weak Precision Tests (EWPT). From these analyses, we will obtain an upper limit on the kinetic mixing parameter $\epsilon$ as a function of the hidden gauge boson mass $m_{X}$.

\section{$3.1 g_{\mu}-2$}

The exchange of the hidden gauge boson $X$ induces a contribution to the anomalous magnetic moment of the muon, $a_{\mu}=\left(g_{\mu}-2\right) / 2$. The modified couplings of the $Z$ 
boson induce an additional contribution. Adopting the formula in Ref. [12], we find:

$$
\delta a_{\mu} \approx \frac{\left(g_{\mu V}^{X}\right)^{2}-5\left(g_{\mu A}^{X}\right)^{2}}{12 \pi^{2}} \frac{m_{\mu}^{2}}{m_{X}^{2}}+\Delta \frac{\left(g_{\mu V}^{Z}\right)^{2}-5\left(g_{\mu A}^{Z}\right)^{2}}{12 \pi^{2}} \frac{m_{\mu}^{2}}{m_{Z}^{2}}
$$

where $g_{\mu V, A}^{X, Z} \equiv g_{\mu R}^{X, Z} \pm g_{\mu L}^{X, Z}$ are given in Appendix $\mathrm{A}$ and $\Delta$ represents the deviation from the value calculated in the SM due to the modification of $Z$ couplings. The difference between the improved Standard Model (SM) prediction of $a_{\mu}$ and the latest experimental value for $a_{\mu}$ is 19

$$
\delta a_{\mu}=(31.6 \pm 7.9) \cdot 10^{-10} .
$$

In Fig. 1, we present the contribution to $a_{\mu}$ from the $X$ gauge boson and the modified $Z$ couplings as a function of $m_{X}$ for various values of $\sin \epsilon$ in the ranges $m_{X}<m_{Z}$ (upper panel) and $m_{X}>m_{Z}$ (lower panel). As shown in Fig. 1, the contribution from our model is well below the current limit with the exception of very small $m_{X}$ and sizable $\sin \epsilon$.

\subsection{Parity-violation effect in atomic physics}

The strength of the vector part of the $Z$ weak neutral current (i.e. the weak force) between interacting quarks and leptons can be characterized by their weak charge. This weak charge governs parity-violation effect in atomic physics. The deviation of the present experimental results on the weak charge for cesium from the theoretical SM predictions corresponds to an uncertainty of less than 1\%. Consequently, the parity-violation effect in atomic physics can provide strong constraints for low $m_{X}$ [11, 12], if the new effect from the couplings of the $Z$ and $X$ gauge bosons to electrons and quarks violates parity. Adopting the result in Ref. [12], the limit on the product of the axial coupling to the electron and its (average) vector coupling is given by

$$
-1.5 \times 10^{-8} \mathrm{GeV}^{-2} \lesssim \mathrm{APV} \equiv \frac{g_{e A}^{X} g_{q V}^{X}}{m_{X}^{2}}+\Delta \frac{g_{e A}^{Z} g_{q V}^{Z}}{m_{Z}^{2}} \lesssim 0.6 \times 10^{-8} \mathrm{GeV}^{-2},
$$

where $g_{f V}^{Z, X}=\left(g_{f L}^{Z, X}+g_{f R}^{Z, X}\right) / 2$ and $g_{f A}^{Z, X}=\left(g_{f L}^{Z, X}-g_{f R}^{Z, X}\right) / 2$, and $\Delta$ again represents the deviation from the Standard Model value. Fig. 2 shows the atomic parity-violation (APV) effect from the $X$ gauge boson and the modified $Z$ couplings as a function of $m_{X}$ for $\sin \epsilon=0.03,0.02,0.01$, and 0.005 in the range $m_{X}<m_{Z}$ (upper panel), and $\sin \epsilon=0.4,0.2,0.1$, and 0.05 in the range $m_{X}>m_{Z}$ (lower panel).

\section{$3.3 \rho$ parameter}

As mentioned in Section 2, the $\rho$ parameter is defined as:

$$
\rho=\frac{m_{W}^{2}}{m_{Z}^{2} c_{W}^{2}}=\frac{s_{W}^{2}}{s_{\hat{W}}^{2}},
$$



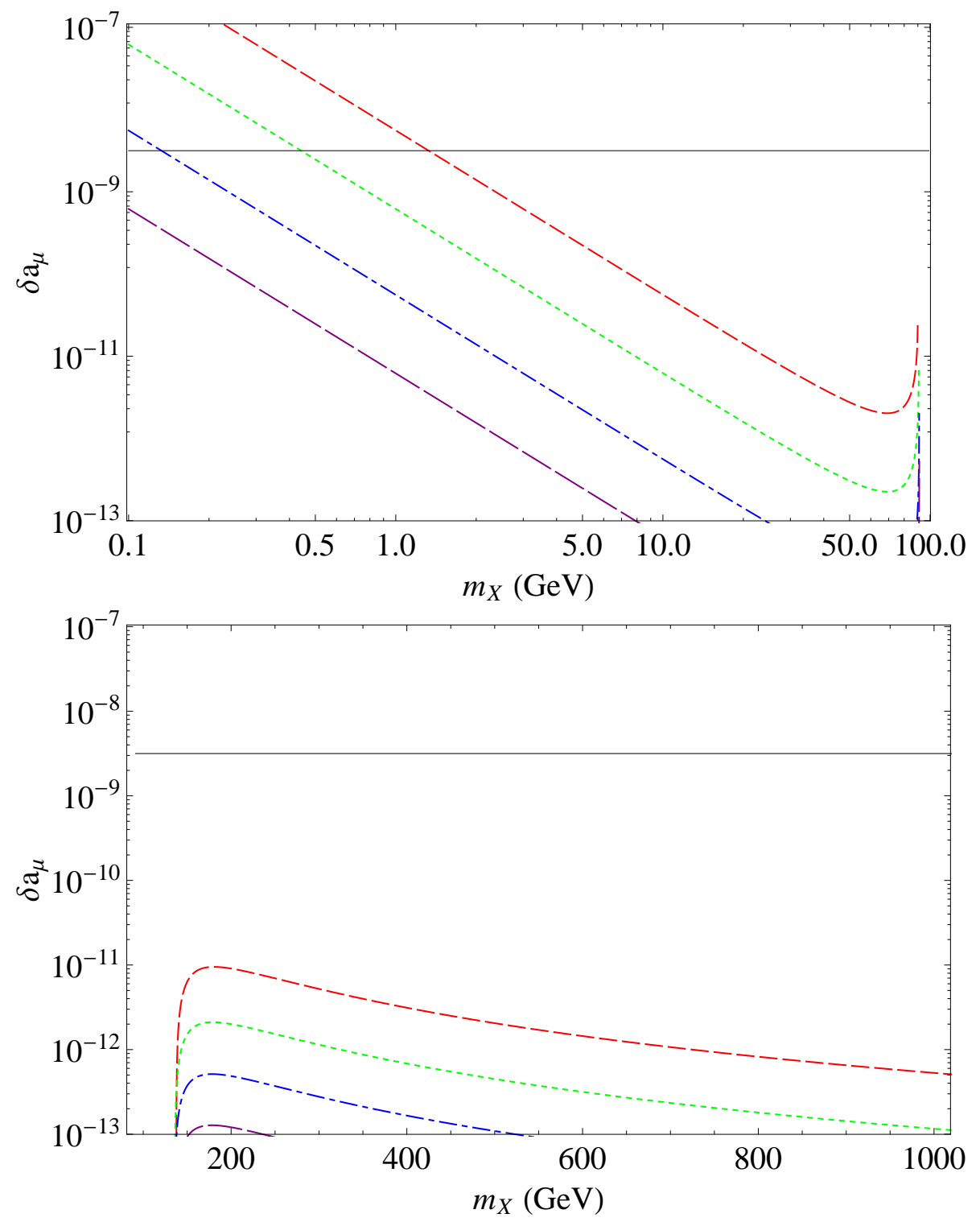

Figure 1: Contribution to the anomalous magnetic moment of the muon, $\delta a_{\mu}$ from the hidden $U(1)_{X}$ model. In the upper panel, the red dashed, green dotted, blue dot-dashed, and purple long-dashed lines show the four cases $\sin \epsilon=0.03,0.01,0.003$, and 0.001 for $m_{X}<m_{Z}$; in the lower panel, the same line-styles and colors show $\sin \epsilon=0.4,0.2,0.1$, and 0.05 for $m_{X}>m_{Z}$. The horizontal solid line is the current limit on the difference between the SM prediction and the latest experimental value [19].

and the deviation of $\rho$ from $1, \hat{\delta} \equiv \rho-1$, is determined by $\sin \epsilon$ according to Eq. (2.18). Therefore, the global fit for the $\rho$ parameter, $\rho-1=4_{-4}^{+8} \times 10^{-4}$ [18], results in a limit on the parameters $\epsilon$ and $m_{X}$. In Fig. 3, we present the deviation of the $\rho$ parameter as a function of $m_{X}$ for $\sin \epsilon=0.1,0.03,0.01$, and 0.003 in the range $m_{X}<m_{Z}$ 

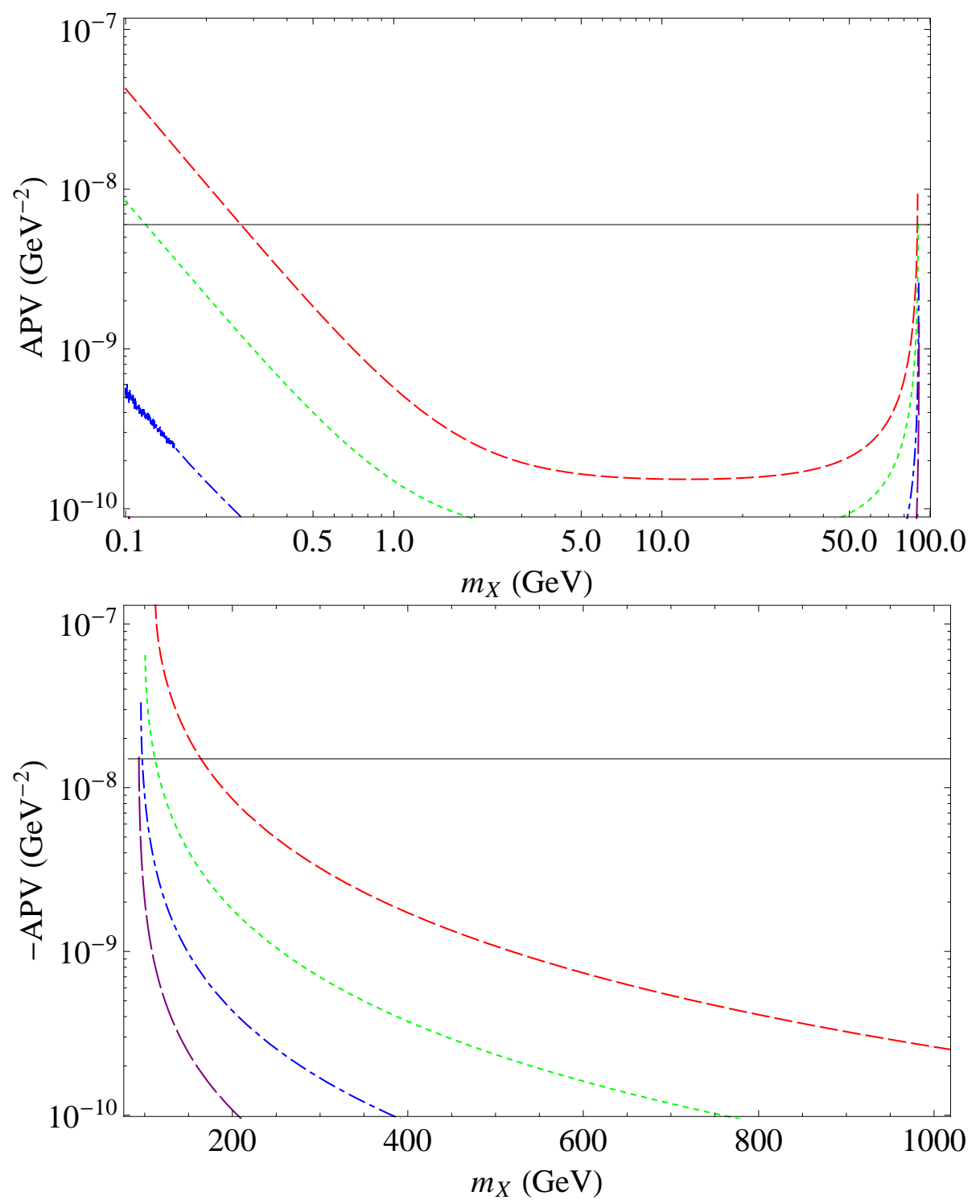

Figure 2: Atomic parity-violation (APV) effect from the hidden $U(1)_{X}$ model. In the upper panel, the red dashed, green dotted, blue dot-dashed, and purple long-dashed lines show the APV effect corresponding to $\sin \epsilon=0.03,0.02,0.01$, and 0.005 for $m_{X}<m_{Z}$; in the lower panel, the same line-styles and colors show $\sin \epsilon=0.4,0.2,0.1$, and 0.05 for $m_{X}>m_{Z}$. The horizontal solid line represents the upper bound given by the difference between the experimental values on the weak charge of cesium and the SM predictions [11].

(upper panel) and for $\sin \epsilon=0.4,0.2,0.1$, and 0.05 in the range $m_{X}>m_{Z}$ (lower panel). This constraint is stronger than limits from the $g_{\mu}-2$ and the APV effect discussed in the previous Sections. The upper bound on $\sin \epsilon$ from the $\rho$ parameter is shown as a function of $m_{X}$ in Fig. 1 . To obtain this bound we use the $2 \sigma$ limit on the $\rho$ parameter. 

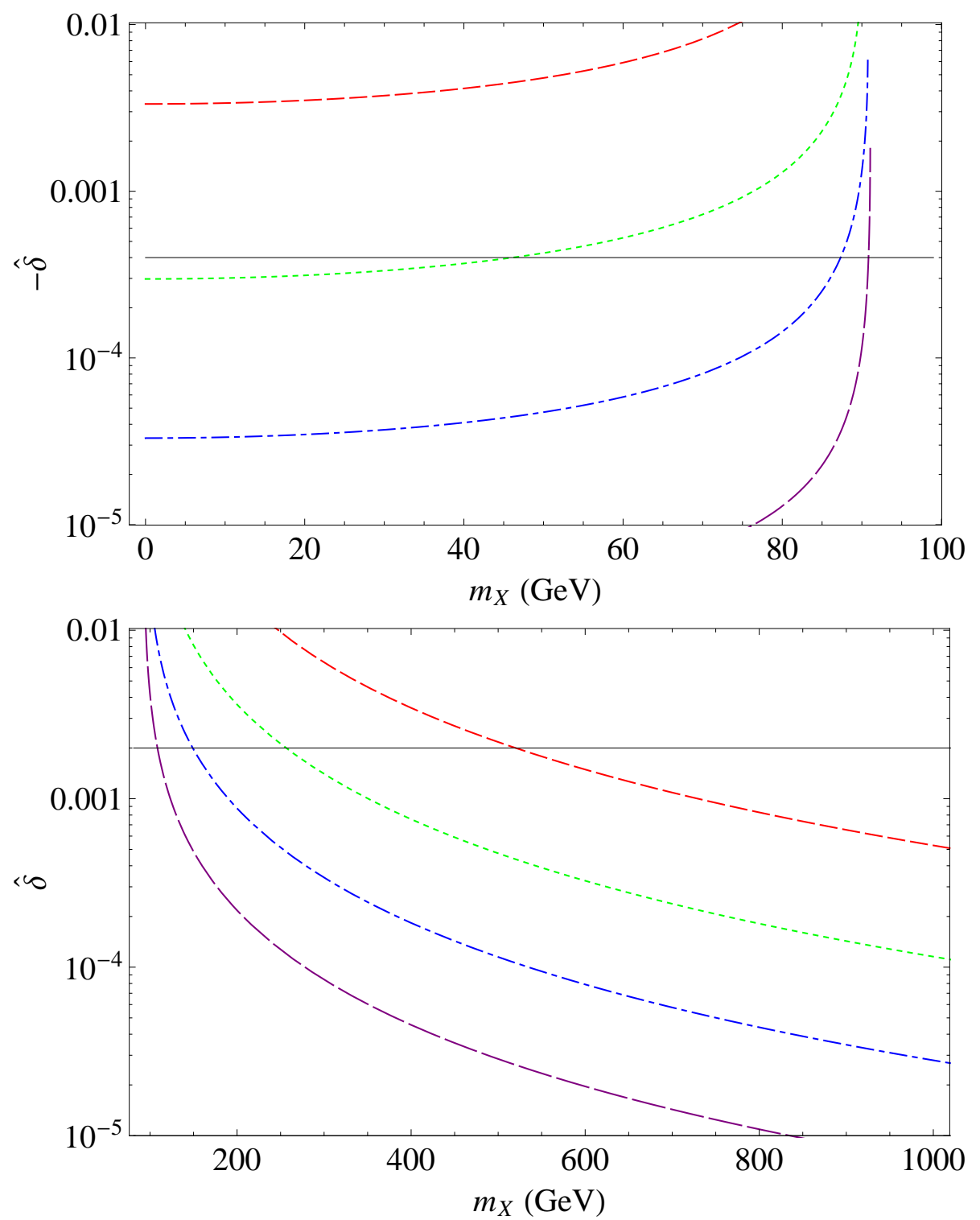

Figure 3: Difference from unity of the $\rho$ parameter, $\hat{\delta}=\rho-1$, due to the hidden $U(1)_{X}$ model. In the upper panel, the deviation of $\rho$ is shown for $\sin \epsilon=0.1,0.03,0.01$, and 0.003 as red dashed, green dotted, blue dot-dashed, and purple long-dashed lines, respectively, and for the case $m_{X}<m_{Z}$. In the lower panel, the same line-styles and colors show the cases $\sin \epsilon=0.4,0.2,0.1$, and 0.05 for $m_{X}>m_{Z}$. The solid horizontal line shows the $2 \sigma$ limit from the global fit [18].

\subsection{EWPT}

The constraints on the hidden $U(1)_{X}$ model from electroweak precision tests (EWPT) have been analyzed in Refs. [13, 14]. Here, we adopt the result of Ref. [13] which 


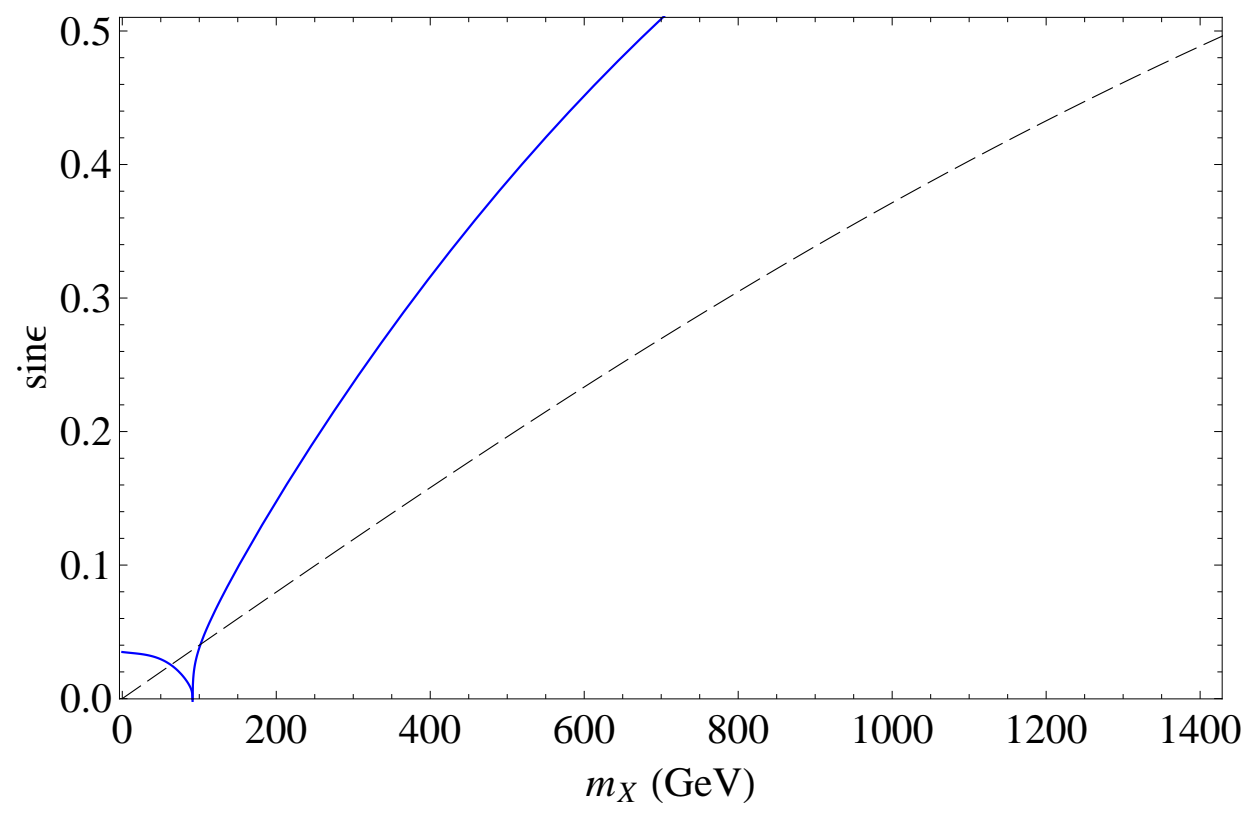

Figure 4: Upper bounds on the kinetic mixing parameter $\sin \epsilon$. The solid line shows the limit from the $\rho$ parameter [18], while the dashed line shows the limit from EWPT [13].

puts a conservative limit:

$$
\left(\frac{\tan \epsilon}{0.1}\right)^{2}\left(\frac{250 \mathrm{GeV}}{m_{X}}\right)^{2} \lesssim 1
$$

This limit is more stringent than that from the $\rho$ parameter except for $m_{X}$ around $m_{Z}$. The $\rho$ parameter and EWPT bounds in the sin $\epsilon-m_{X}$ plane are shown in Fig. 4 .

\section{Thermal relic abundance of dark matter}

The relic abundance of the DM candidate $\psi$ depends on the $\psi \bar{\psi}$ annihilation cross section to Standard Model particles, which proceeds through $s$-channel exchange of $Z$ and $X$ bosons in the zero-velocity limit. In particular, the annihilation modes include $\psi \bar{\psi} \rightarrow f \bar{f}, W^{+} W^{-}, X h$, and $Z h$, which give the following annihilation cross 
section times velocity:

$$
\begin{aligned}
\left\langle\sigma_{A} v\right\rangle \simeq & \sum_{f} \frac{N_{f}}{2 \pi} m_{\psi}^{2} \sqrt{1-\frac{m_{f}^{2}}{m_{\psi}^{2}}}\left[\left(G_{f L}^{2}+G_{f R}^{2}\right)\left(1-\frac{m_{f}^{2}}{4 m_{\psi}^{2}}\right)+\frac{3}{2} G_{f L} G_{f R} \frac{m_{f}^{2}}{m_{\psi}^{2}}\right] \\
& +\frac{1}{\pi} m_{\psi}^{2} G_{W}^{2}\left(1-\frac{m_{W}^{2}}{m_{\psi}^{2}}\right)^{3 / 2}\left[\frac{m_{\psi}^{4}}{m_{W}^{4}}+5 \frac{m_{\psi}^{2}}{m_{W}^{2}}+\frac{3}{4}\right] \\
& +\frac{1}{8 \pi} G_{X h}^{2} \sqrt{1-\frac{m_{Z}^{2}}{m_{\psi}^{2}}+\left(\frac{\Delta m_{h Z}^{2}}{4 m_{\psi}^{2}}\right)^{2}}\left\{1+\frac{1}{2} \frac{m_{\psi}^{2}}{m_{Z}^{2}}\left[1-\frac{\Delta m_{h Z}^{2}}{2 m_{\psi}^{2}}+\left(\frac{\Delta m_{h Z}^{2}}{4 m_{\psi}^{2}}\right)^{2}\right]\right\} \\
& +\frac{1}{8 \pi} G_{Z h}^{2} \sqrt{1-\frac{m_{X}^{2}}{m_{\psi}^{2}}+\left(\frac{\Delta m_{h X}^{2}}{4 m_{\psi}^{2}}\right)^{2}}\left\{1+\frac{1}{2} \frac{m_{\psi}^{2}}{m_{X}^{2}}\left[1-\frac{\Delta m_{h X}^{2}}{2 m_{\psi}^{2}}+\left(\frac{\Delta m_{h X}^{2}}{4 m_{\psi}^{2}}\right)^{2}\right]\right\}
\end{aligned}
$$

where:

$$
\begin{aligned}
G_{f L} & =\frac{g_{\psi}^{Z} g_{f L}^{Z}}{4 m_{\psi}^{2}-m_{Z}^{2}}+\frac{g_{\psi}^{X} g_{f L}^{X}}{4 m_{\psi}^{2}-m_{X}^{2}} \\
G_{f R} & =\frac{g_{\psi}^{Z} g_{f R}^{Z}}{4 m_{\psi}^{2}-m_{Z}^{2}}+\frac{g_{\psi}^{X} g_{f R}^{X}}{4 m_{\psi}^{2}-m_{X}^{2}} \\
G_{W} & =\frac{g_{\psi}^{Z} g_{W}^{Z}}{4 m_{\psi}^{2}-m_{Z}^{2}}+\frac{g_{\psi}^{X} g_{W}^{X}}{4 m_{\psi}^{2}-m_{X}^{2}} \\
G_{V h} & =\frac{g_{\psi}^{V} g_{X Z}^{h}}{4 m_{\psi}^{2}-m_{V}^{2}}
\end{aligned}
$$

Here, we define $\bar{m}_{V}^{2}=\left(m_{h}^{2}+m_{V}^{2}\right) / 2$ and $\Delta m_{h V}^{2}=m_{h}^{2}-m_{V}^{2}$. For the annihilation to $h Z(h X)$ through $s$-channel exchange of the $X(Z)$ boson, $\left(\Delta m_{h V}^{2} / 4 m_{\psi}^{2}\right)^{2} \ll 1$ is assumed. In the evaluations of the following Sections, we will assume the numerical value $m_{h}=115 \mathrm{GeV}$.

The present relic density of a hidden Dirac fermion $\psi$ can be calculated from the following analytic formula:

$$
\Omega_{\psi} h^{2} \approx 2 \times \frac{1.07 \times 10^{9} \mathrm{GeV}^{-1}}{M_{p l}} \frac{x_{F}}{\sqrt{g_{*}}} \frac{1}{\left\langle\sigma_{A} v\right\rangle},
$$

where $g_{*}$ is the number of relativistic degrees of freedom at the freeze-out temperature $T_{F}$ and $x_{F} \equiv m_{\psi} / T_{F}$ [20]. The extra factor of two on the right-hand side of Eq. (4.3) results from the fact that the annihilation can only occur between particle and antiparticle, since the dark matter candidate is a Dirac fermion. In the following, we will use the $\psi$ relic abundance to constrain our parameter space. In particular, the annihilation cross section $\left\langle\sigma_{A} v\right\rangle$ given in Eq. (4.1) is dominated either by the coupling $G_{X h}$ in the $X h$ final state channel $\left(m_{X} \lesssim 120 \mathrm{GeV}\right)$ or by the couplings 
$G_{f L}$ and $G_{f R}$ in the $f \bar{f}$ final state. One can see from Eq.(A.1) that in both cases $\left\langle\sigma_{A} v\right\rangle$ depends on the parameters $g_{X}$ and $\epsilon$ through the same multiplicative factor, $g_{X} s_{\xi} / c_{\epsilon}$. For each dark matter mass $m_{\psi}$ and hidden gauge boson mass $m_{X}$, we will, therefore, determine the combination $g_{X} s_{\xi} / c_{\epsilon}$ by imposing the recent bound on the dark matter relic density, $\left(\Omega_{\mathrm{DM}} h^{2}\right)_{\text {obs }} \simeq 0.1123$ [21]. Moreover, by fixing $\epsilon$ to its experimental upper bound, the same combination will be used in Section 6 to determine the remaining coupling $g_{X}$.

\section{Direct detection of dark matter}

Satisfying all the previous constraints, we now discuss prospect of observing DM in direct detection experiments. The dark matter particle $\psi$ can elastically scatter off a nucleus through $t$-channel $X$ and $Z$ gauge boson exchange. The spin-independent (SI) DM-nucleon scattering cross section can be calculated from the following effective operator:

$$
\begin{gathered}
\mathcal{L}_{e f f}=b_{f} \bar{\psi} \gamma_{\mu} \psi \bar{f} \gamma^{\mu} f, \\
\text { where } \quad b_{f}=\frac{g_{\psi}^{Z}\left(g_{f L}^{Z}+g_{f R}^{Z}\right)}{2 m_{Z}^{2}}+\frac{g_{\psi}^{X}\left(g_{f L}^{X}+g_{f R}^{X}\right)}{2 m_{X}^{2}} .
\end{gathered}
$$

From the interaction couplings presented in Section 2, one calculates $b_{u}$ and $b_{d}$ to get

$$
\begin{aligned}
b_{p}=+\frac{\hat{g} g_{X}}{4 c_{\hat{W}}} \frac{c_{\xi}^{2}}{c_{\epsilon}} \frac{t_{\xi}}{m_{Z}^{2}}\left[\left(1-4 s_{\hat{W}}^{2}\right)\left(1-\frac{1}{r_{X}}\right)-3 s_{\hat{W}} \frac{t_{\epsilon}}{t_{\xi}}\left(t_{\xi}^{2}+\frac{1}{r_{X}}\right)\right] \\
\simeq \frac{e g_{X}}{4 c_{W} s_{W}} \frac{c_{\xi}^{2}}{c_{\epsilon}} \frac{t_{\xi}}{m_{Z}^{2}}\left\{\left(1-4 s_{W}^{2}\right)\left(1-\frac{1}{r_{X}}\right)-\frac{3}{r_{X}} \frac{s_{W} t_{\epsilon}}{t_{\xi}}\right. \\
\left.\quad-\omega\left[3+\left(1-\frac{1}{r_{X}}\right)\left(\frac{1}{2}+2 s_{W}^{2} \frac{1+t_{W}^{2}}{1-t_{W}^{2}}\right)-\frac{1}{r_{X}} \frac{s_{W} t_{\epsilon}}{t_{\xi}} \frac{3 t_{W}^{2}}{2-2 t_{W}^{2}}\right]\right\}, \\
b_{n}=-\frac{\hat{g} g_{X}}{4 c_{\hat{W}}} \frac{c_{\xi}^{2}}{c_{\epsilon}} \frac{t_{\xi}}{m_{Z}^{2}}\left[\left(1-\frac{1}{r_{X}}\right)+s_{\hat{W}} \frac{t_{\epsilon}}{t_{\xi}}\left(t_{\xi}^{2}+\frac{1}{r_{X}}\right)\right] \\
\simeq-\frac{e g_{X}}{4 c_{W} s_{W}} \frac{c_{\xi}^{2}}{c_{\epsilon}} \frac{t_{\xi}}{m_{Z}^{2}}\left\{\left[1-\frac{1}{r_{X}}\left(1-s_{W} \frac{t_{\epsilon}}{t_{\xi}}\right)\right]+\frac{\omega}{2}\left[1+\frac{1}{r_{X}}\left(1+\frac{s_{W} t_{W}^{2} t_{\epsilon}}{\left(1-t_{W}^{2}\right) t_{\xi}}\right)\right]\right\} .
\end{aligned}
$$

For a given nucleus ${ }_{Z}^{A} N$, one has $b_{N}=Z b_{p}+(A-Z) b_{n}$. Finally, the $\psi$-nucleon elastic scattering cross section is given by [22]:

$$
\sigma_{n, p}=\frac{1}{64 \pi} \frac{\mu_{n, p}^{2}}{A^{2}} b_{N}^{2},
$$

where $\mu_{n, p}$ is the DM-nucleon reduced mass. In Fig. 5, we present the SI DM-nucleon scattering cross section as a function of $m_{X}$ for $m_{\psi}=50,100,300$, and $1000 \mathrm{GeV}$. From this figure, one can see that small DM masses around $m_{\psi} \sim 100 \mathrm{GeV}$ are at the level of the current sensitivity of direct detection experiments. On the other hand, 


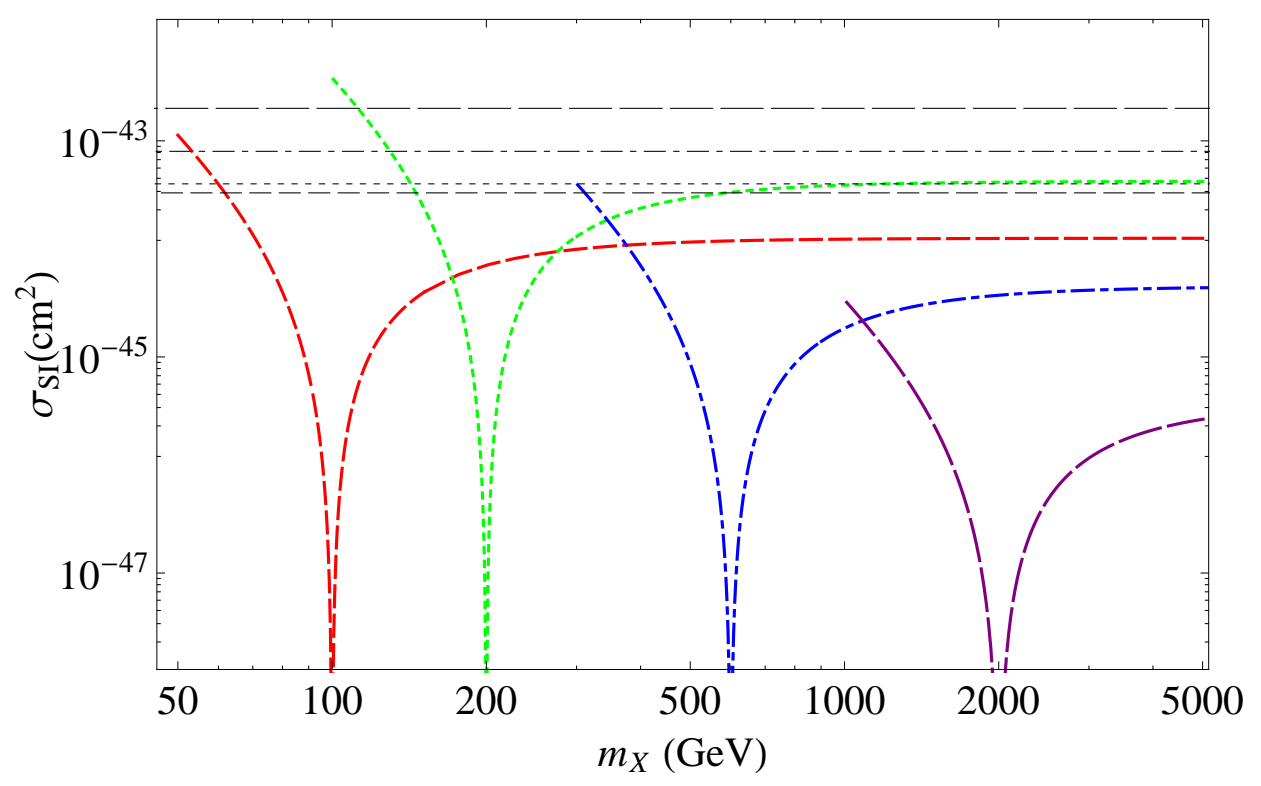

Figure 5: Spin-independent DM-nucleon scattering cross section $\sigma_{\mathrm{SI}}$ calculated by normalizing the DM-nucleon couplings to the values that provide the observed DM relic density (see text). The red dashed, green dotted, blue dot-dashed, and purple long-dashed lines correspond to $m_{\psi}=50,100,300$, and $1000 \mathrm{GeV}$, respectively. The experimental limits from CDMS II [15] corresponding to $m_{\psi}=100,300$, and $1000 \mathrm{GeV}$ are shown by the dotted, dot-dashed, and long-dashed lines, respectively . The experimental limit for $m_{\psi}=50$, which is taken from XENON100 [16], is shown by the dashed line.

for a larger mass up to around $m_{\psi} \sim 300 \mathrm{GeV}$ and smaller $m_{X}$, DM signals can be detectable in the near future.

To calculate the SI DM-nucleon scattering cross sections shown in Fig. 5, we have normalized the $b_{f}$ coefficients given in Eq. (5.2) by using the relic abundance constraint. Notice that, as in the case of the annihilation cross section, also $b_{f}$ depends on $g_{X}$ and $\epsilon$ through the multiplicative factor $g_{X} s_{\xi} / c_{\epsilon}$. An important consequence of this is that, at fixed values of $m_{X}$ and $m_{\psi}$, the dependence on $g_{X}$ and $\epsilon$ cancels out in the ratio $\sigma_{n, p} /\left\langle\sigma_{A} v\right\rangle$. This implies that in our model the direct detection cross section $\sigma_{n, p}$ is potentially able to put robust constraints on $m_{X}$ and $m_{\psi}$, once the $\psi$ particle is assumed to provide the observed DM relic density in the Universe. On the other hand, this degeneracy in $g_{X}$ and $\epsilon$ is not present in the calculation of accelerator signals, as will be discussed in the next Section.

\section{Tevatron and LHC probes of $\mathrm{U}(1)_{X}$}

For the analysis of collider searches we concentrate on the dimuon signal from production and decay of the hidden gauge boson $X$ at the Tevatron and LHC. For this, 
we need to calculate the corresponding branching ratios, which depend on the kinematically available decay channels. The relevant decay rates of the $X$ gauge boson are given by:

$$
\begin{gathered}
\Gamma(X \rightarrow f \bar{f})=\frac{N_{f}}{12 \pi} \sqrt{\frac{m_{X}^{2}}{4}-m_{f}^{2}}\left\{\left[\left(g_{f L}^{X}\right)^{2}+\left(g_{f R}^{X}\right)^{2}\right]\left(1-\frac{m_{f}^{2}}{m_{X}^{2}}\right)+6\left(g_{f L}^{X}\right)^{2}\left(g_{f R}^{X}\right)^{2} \frac{m_{f}^{2}}{m_{X}^{2}}\right\}, \\
\Gamma(X \rightarrow \psi \bar{\psi})=\frac{\left(g_{\psi}^{X}\right)^{2}}{6 \pi} \sqrt{\frac{m_{X}^{2}}{4}-m_{\psi}^{2}}\left(1+2 \frac{m_{\psi}^{2}}{m_{X}^{2}}\right), \\
\Gamma(X \rightarrow h Z)=\frac{\left(g_{X Z}^{h}\right)^{2}}{48 \pi m_{X}^{3}} \sqrt{\left[m_{X}^{2}-\left(m_{Z}+m_{h}\right)^{2}\right]\left[m_{X}^{2}-\left(m_{Z}-m_{h}\right)^{2}\right]} \\
\times\left[2+\frac{1}{2}\left(\frac{m_{X}}{m_{Z}}+\frac{m_{Z}}{m_{X}}-\frac{m_{h}^{2}}{m_{X} m_{Z}}\right)\right] \\
\Gamma\left(X \rightarrow W^{+} W^{-}\right)=\frac{\left(g_{W}^{X}\right)^{2}}{192 \pi} m_{X}\left(\frac{m_{X}}{m_{W}}\right)^{4}\left(1-4 \frac{m_{W}^{2}}{m_{X}^{2}}\right)^{3 / 2}\left[1+20 \frac{m_{W}^{2}}{m_{X}^{2}}+12 \frac{m_{W}^{4}}{m_{X}^{4}}\right](6.1)
\end{gathered}
$$

We wish now to combine the constraints summarized in the previous Sections to put bounds on the parameter space of our model, spanned by the four parameters $m_{X}, m_{\psi}, g_{X}$, and $\sin \epsilon$. In particular, the bounds which turn out to be the most constraining, and that will be the most relevant for the present discussion are: i) the upper bound on $\sin \epsilon$ from EWPT discussed in Section 3.4; ii) the condition that the $\psi$ relic density $\Omega_{\psi} h^{2}$ is equal to the observed dark matter relic density value $\left(\Omega_{\mathrm{DM}} h^{2}\right)_{\text {obs }} \simeq 0.1123$; iii) the upper bound on $\sigma(p \bar{p} \rightarrow X) B R(X \rightarrow \mu \bar{\mu})$ from CDF which will be discussed below in this Section. As a first approach to this problem, we fix $\sin \epsilon$ to its EWPT upper bound and $g_{X}$ to the value required to provide $\left(\Omega_{\mathrm{DM}} h^{2}\right)_{\text {obs }} \simeq 0.1123$ and calculate the expected number of dimuon events at the Tevatron. The result of this exercise is shown in Fig. 6, where the curves show the quantity $\sigma(p \bar{p} \rightarrow X) B R(X \rightarrow \mu \bar{\mu})$ as a function of $m_{X}$ at fixed values of $m_{\psi}$. Here and in the following, we calculate the production cross section $\sigma(p \bar{p} \rightarrow X)$ by making use of the PYTHIA code [23]. In the same Figure, the shaded area shows the upper bound from CDF on the same quantity [24]. From this Figure, one can see that, depending on the value of $m_{\psi}$, the expected number of dimuon events at the Tevatron $\sigma(p \bar{p} \rightarrow X) B R(X \rightarrow \mu \bar{\mu})$ can exceed the CDF upper bound.

This is studied in detail in Fig. 7 in the $m_{X}-m_{\psi}$ plane. In this figure, the region to the left of the black solid line boundary is excluded by the CDF upper bound. In particular, one can see from this plot that the range $m_{X} \lesssim 600 \mathrm{GeV}$ is excluded unless $m_{\psi} \lesssim 200 \mathrm{GeV}$. In fact, in this latter case the process $p \bar{p} \rightarrow X \rightarrow \mu \bar{\mu}$ at the Tevatron can be suppressed weakening the CDF bound because the invisible decay 


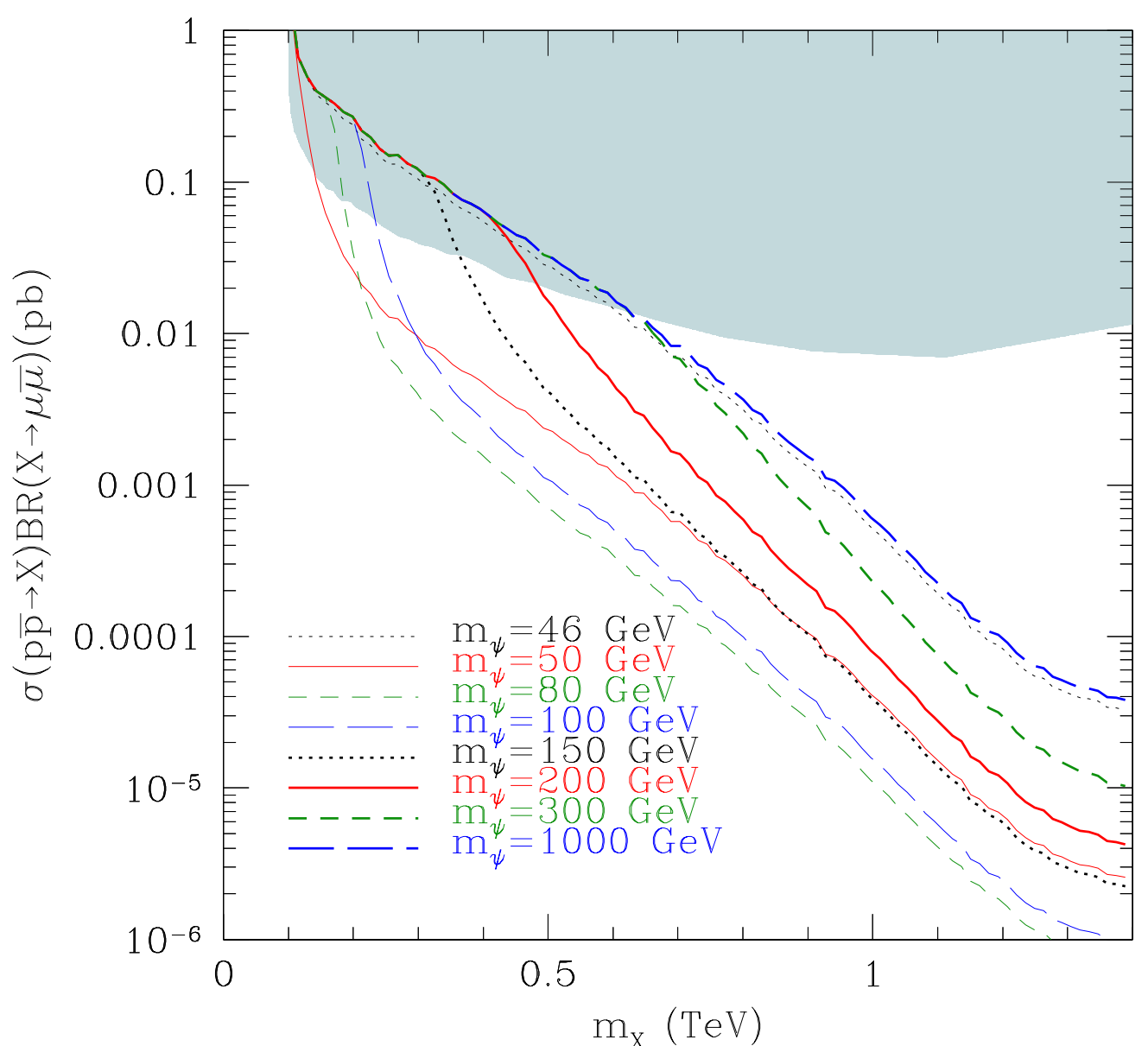

Figure 6: Expected number of events for the production of the $X$ boson and its decay to $\mu \bar{\mu}$ at Tevatron, calculated for different values of $m_{\psi}$. The shaded area is excluded by dimuon searches at CDF [24]. Notice that the curve for $m_{\psi}=1000 \mathrm{GeV}$ is cut at $m_{X} \sim$ $360 \mathrm{GeV}$ due to the perturbativity bound shown as a shaded area in Fig. 0 .

channel $X \rightarrow \psi \bar{\psi}$ can become sizable. An exception to this is when $m_{\psi} \simeq m_{Z} / 2$, because in this case the resonant annihilation of $\psi$ particles in the calculation of the relic density requires very low values of the coupling $g_{X}$ in order to provide the correct amount dark matter. As a consequence of this, one has $B R(X \rightarrow \psi \bar{\psi}) \simeq 0$ and the constraint on $m_{X}$ jumps to the same value $m_{X} \lesssim 600 \mathrm{GeV}$ that one finds for higher values of $m_{\psi}$. However, in Fig. 7, the values of $m_{X}$ and $m_{\psi}$ to the left of the solid line boundary are only excluded if $\sin \epsilon$ is fixed to its EWPT upper bound. Consequently, in this region of the $m_{X}-m_{\psi}$ parameter space, the quantity $\sigma(p \bar{p} \rightarrow X) B R(X \rightarrow \mu \bar{\mu})$ can be brought below the CDF constraint by using a value for $\sin \epsilon$ smaller than the EWPT bound. In other words, Fig. 7 shows that for $m_{X} \lesssim$ $600 \mathrm{GeV}$ the CDF constraint can be stronger than the EWPT bound.

In order to see the combined effect of the two constraints on the parameter space of our model, we provide Fig. 8 where we show the upper bound on $\sin \epsilon$ as a function of $m_{X}$ for different fixed values of $m_{\psi}$ when the CDF constraint is combined to the 


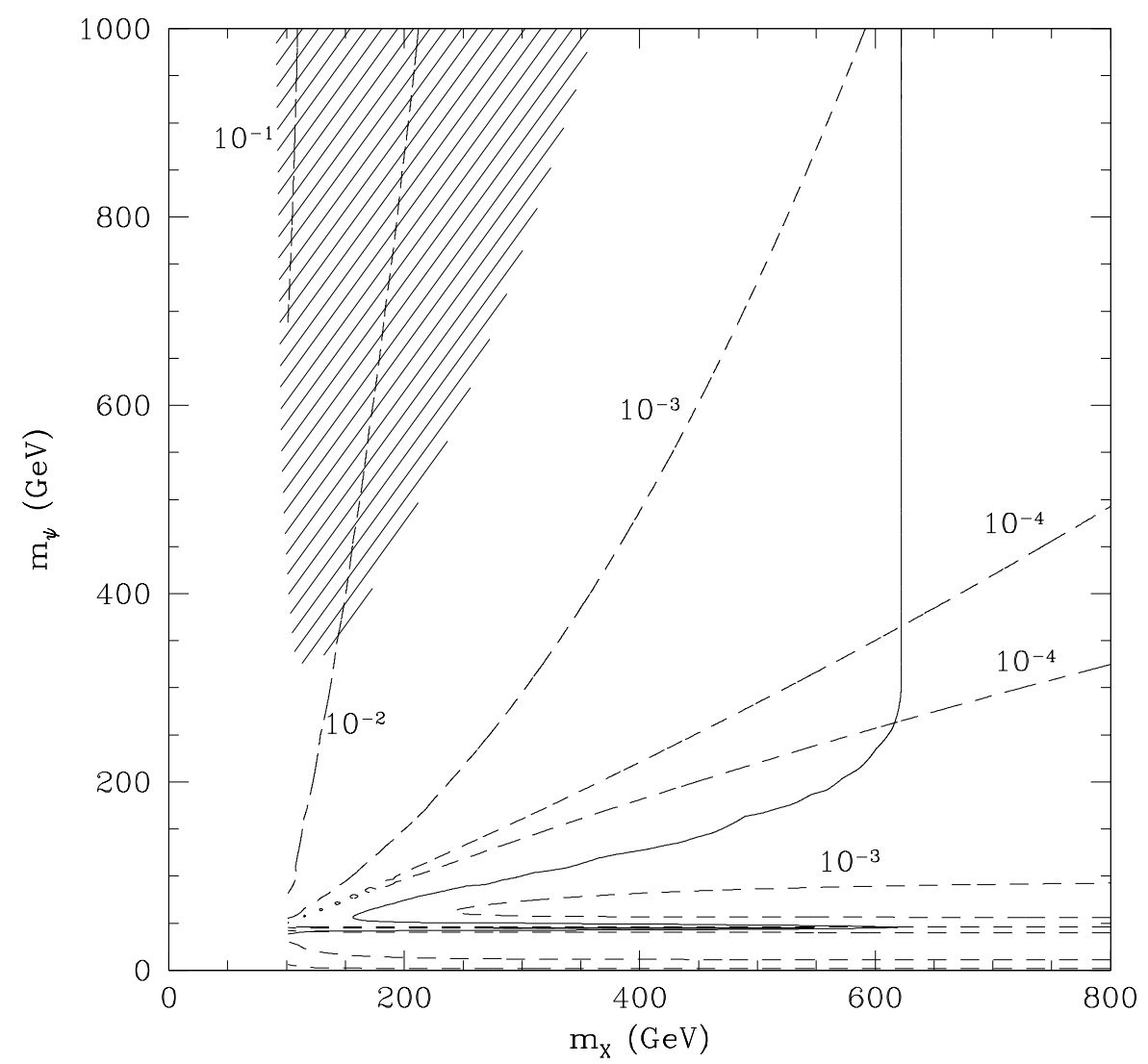

Figure 7: The region to the left of the solid line is excluded by CDF dimuon $X$ searches in the $m_{X}-m_{\psi}$ plane when the $\sin \epsilon$ parameter is set to its upper bound from EWPT. The dashed lines show the contour plots in the same plane of the minimal values of $\sin \epsilon$ compatible to the cosmological constraint and with the perturbativity requirement $\left(g_{\psi}^{X}\right)^{2} /(4 \pi)<1$. Within the shaded area, the minimal value of $\sin \epsilon$ is already exceeding the upper bound shown in Fig. 8 (see text).

EWPT one. In this Figure, the upper thick solid line shows the constraint from the $\rho$ parameter [18], as discussed in Section 3.3, while the lower thick solid line shows the constraint from EWPT [13], as discussed in Section 3.4. The latter bound is mostly more constraining than the former, and is saturated for $m_{X} \gtrsim 600 \mathrm{GeV}$. On the other hand, the set of curves at $m_{X} \lesssim 600 \mathrm{GeV}$ show how the CDF constraint becomes important at lower $X$ masses for different values of $m_{\psi}$. As already discussed in connection to Figs. 6 and 7, one can see from Fig. 8 that the CDF bound on the parameter space of our model can be stronger than the EWPT one for $m_{X} \lesssim 600$ $\mathrm{GeV}$, with the exception $m_{\psi} \simeq m_{Z} / 2$.

To determine the curves in Fig. 8, the quantity $\sigma(p \bar{p} \rightarrow X) B R(X \rightarrow \mu \bar{\mu})$ has been calculated by fixing $g_{X}$ as before using the relic abundance of the $\psi$ particle. In particular, following this procedure one has to check that the upper bound on $\sin \epsilon$ is consistent to the perturbativity bound $\left(g_{\psi}^{Z}\right)^{2} /(4 \pi),\left(g_{\psi}^{X}\right)^{2} /(4 \pi)<1$. Indeed, for 
some intervals of $m_{X}$ and $m_{\psi}$, the combination of the relic abundance, the CDF, and EWPT constraints require $\left(g_{\psi}^{X}\right)^{2} /(4 \pi)>1$. The corresponding excluded region in the $m_{X}-m_{\psi}$ plane is shown as a shaded area in Fig. 7. In particular, in this region of the parameter space, the $f \bar{f}$-final state contribution to the annihilation cross section $\left\langle\sigma_{A} v\right\rangle$ (which dominates at larger masses) is suppressed, while the upper bound on $\sin \epsilon$ is particularly constraining, driving $g_{\psi}^{X}$ to large values in order to keep $\Omega_{\psi} h^{2}$ equal to the observed cosmological DM abundance.

Note that the shaded area excluded by the perturbativity limit does not extend below $m_{X} \sim 90 \mathrm{GeV}$. This is due to the fact that in this region of the parameter space, where the $f \bar{f}$-final state contribution in $\left\langle\sigma_{A} v\right\rangle$ is suppressed, the $X h$-final state takes over as the dominant channel, thanks to the sizable value of the $t_{\xi}$ parameter in the $g_{X Z}^{h}$ coupling when $m_{X} \sim m_{Z}$ (see Eqs. (2.3) and (A.1)). The ensuing enhancement of $\left\langle\sigma_{A} v\right\rangle$ implies that for $m_{X} \lesssim 90 \mathrm{GeV}$ the observed relic abundance can be obtained for $\left(g_{\psi}^{Z}\right)^{2} /(4 \pi),\left(g_{\psi}^{X}\right)^{2} /(4 \pi)<1$. Notice that, for consistency, the curves in Figs. 6 and 9 for $m_{\psi}=1000 \mathrm{GeV}$ have been cut at $m_{X} \sim 360 \mathrm{GeV}$. Moreover, since at fixed $m_{X}$ and $m_{\psi}$, the cosmological constraint fixes the combination $g_{X} s_{\xi} / c_{\epsilon}$ which vanishes when $\epsilon \rightarrow 0$, keeping the value of $\Omega_{\psi} h^{2}$ fixed when $\epsilon \rightarrow 0$ requires $g_{X} \rightarrow \infty$. So, imposing $\left(g_{\psi}^{Z}\right)^{2} /(4 \pi),\left(g_{\psi}^{X}\right)^{2} /(4 \pi)<1$ allows also to get a lower bound on $\sin \epsilon$ as a function of $m_{X}$ and $m_{\psi}$. The result of this procedure is also shown in Fig.7, where contour plots at fixed values of $(\sin \epsilon)_{\min }$ are plotted as dashed lines in the $m_{X}-m_{\psi}$ plane.

The curves given in Fig. 8 can be used to estimate the perspectives of detection of our model at the LHC. This is shown in Fig. 9, where the quantity $\sigma(p p \rightarrow$ $X) B R(X \rightarrow \mu \bar{\mu})$ at the center-of-mass energy of $14 \mathrm{TeV}$ is shown by a set of lines as a function of $m_{X}$ at fixed values of $m_{\psi}$. In each of these curves, the value of $\sin \epsilon$ is fixed to the upper bound shown in Fig. 8 for the corresponding $m_{\psi}$. The peculiar hollow shape of some of the curves for $m_{X} \lesssim 600 \mathrm{GeV}$ corresponds to the region of the parameter space where the CDF constraint on $\sin \epsilon$ overcomes that from EWPT. In the same Figure, an estimation is also given of the $5 \sigma$ discovery reach at the LHC for an exposure of $10 \mathrm{fb}^{-1}$ [25]. We finally recall once again that, at variance with the analysis of signals at accelerators, the calculation of the $\psi$-nucleon elastic cross section relevant for direct DM searches does not rely on any assumption on $\epsilon$ because at fixed values of $m_{X}$ and $m_{\psi}$ the cross section $\sigma_{n, p}$ is unambiguously determined by the relic abundance.

\section{Conclusions}

We postulated the existence of a hidden $U(1)_{X}$ gauge symmetry and a Dirac dark matter fermion charged under $U(1)_{X}$. Assuming that the hidden sector communicates with the Standard Model sector only through the kinetic mixing between $U(1)_{X}$ and $U(1)_{Y}$, the phenomenology of such scenario depends on four parameters: 


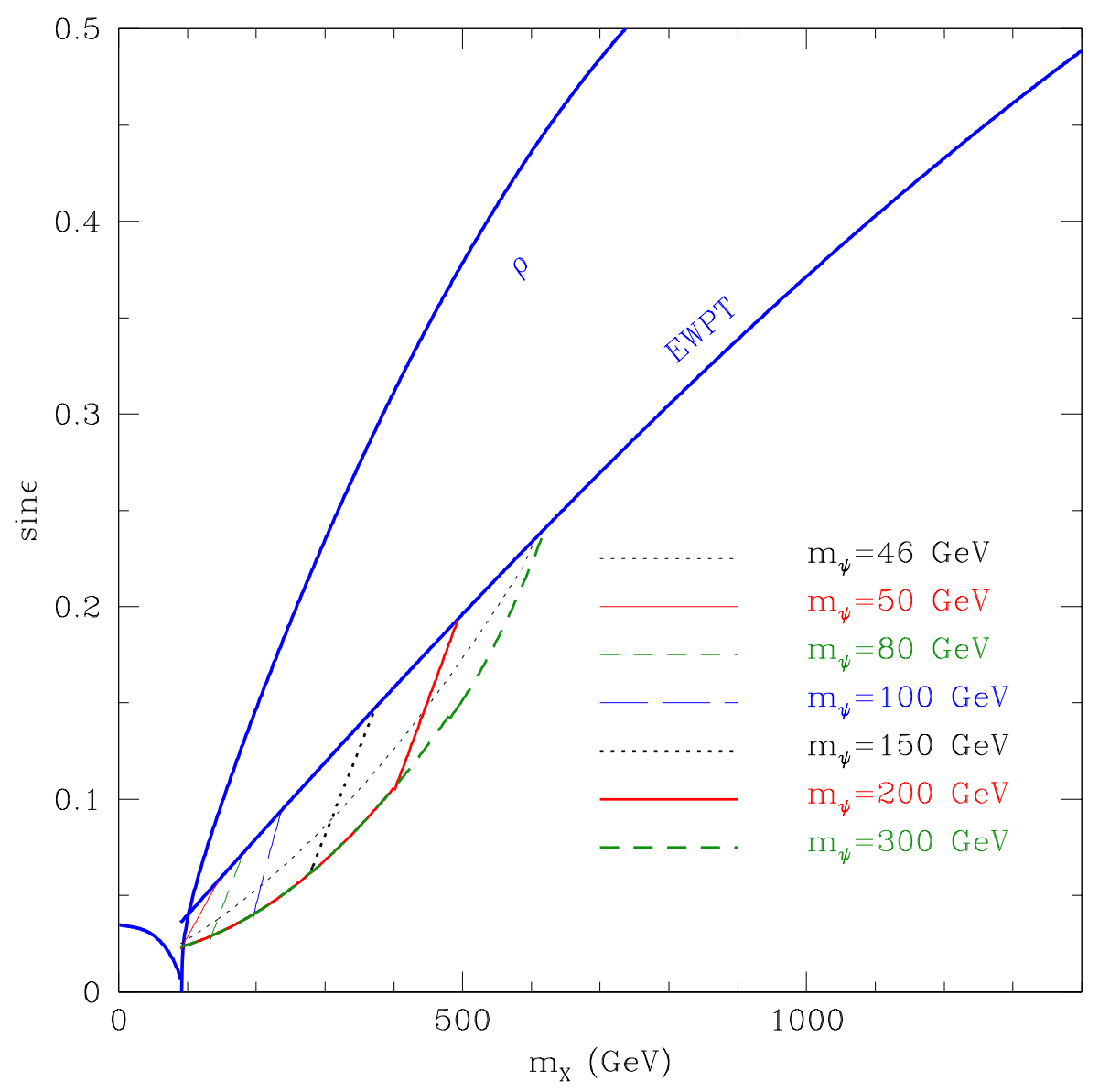

Figure 8: Upper bounds on the $\sin \epsilon$ parameter as a function of $m_{X}$ for different values of $m_{\psi}$. The upper thick solid line shows the constraint from the $\rho$ parameter [18], as discussed in Section 3.3, while the lower thick solid line shows the constraint from EWPT [13], as discussed in Section 3.4. For $m_{X} \lesssim 600 \mathrm{GeV}$, the $\sin \epsilon$ parameter is also constrained by dimuon searches at CDF [24], depending on the value of $m_{\psi}$, as explained in detail in Section 6.

the kinetic mixing angle, the mass of the $X$ boson, the dark matter mass, the coupling between the DM particle and the $X$ gauge boson. We have considered various observables constraining the kinetic mixing parameter and the $U(1)_{X}$ coupling constant as a function of the $X$ gauge boson mass. Since the dark matter annihilation to the Standard Model fields proceeds through kinetic mixing, we have used the relic density of the DM particle (determined by the standard thermal freeze-out process) to constrain a combination of the kinetic mixing and the $X$ gauge boson coupling, requiring $\Omega_{\psi} h^{2}$ to be equal to the observed DM density in the Universe. Saturating all these constraints, we analyzed the spin-independent elastic cross-section of the dark matter off nucleons showing that a large parameter space is within the sensitivity of future direct detection experiments. We have also analyzed collider searches of the $X$ gauge boson, concentrating on the dimuon signal at the Tevatron and the LHC. 


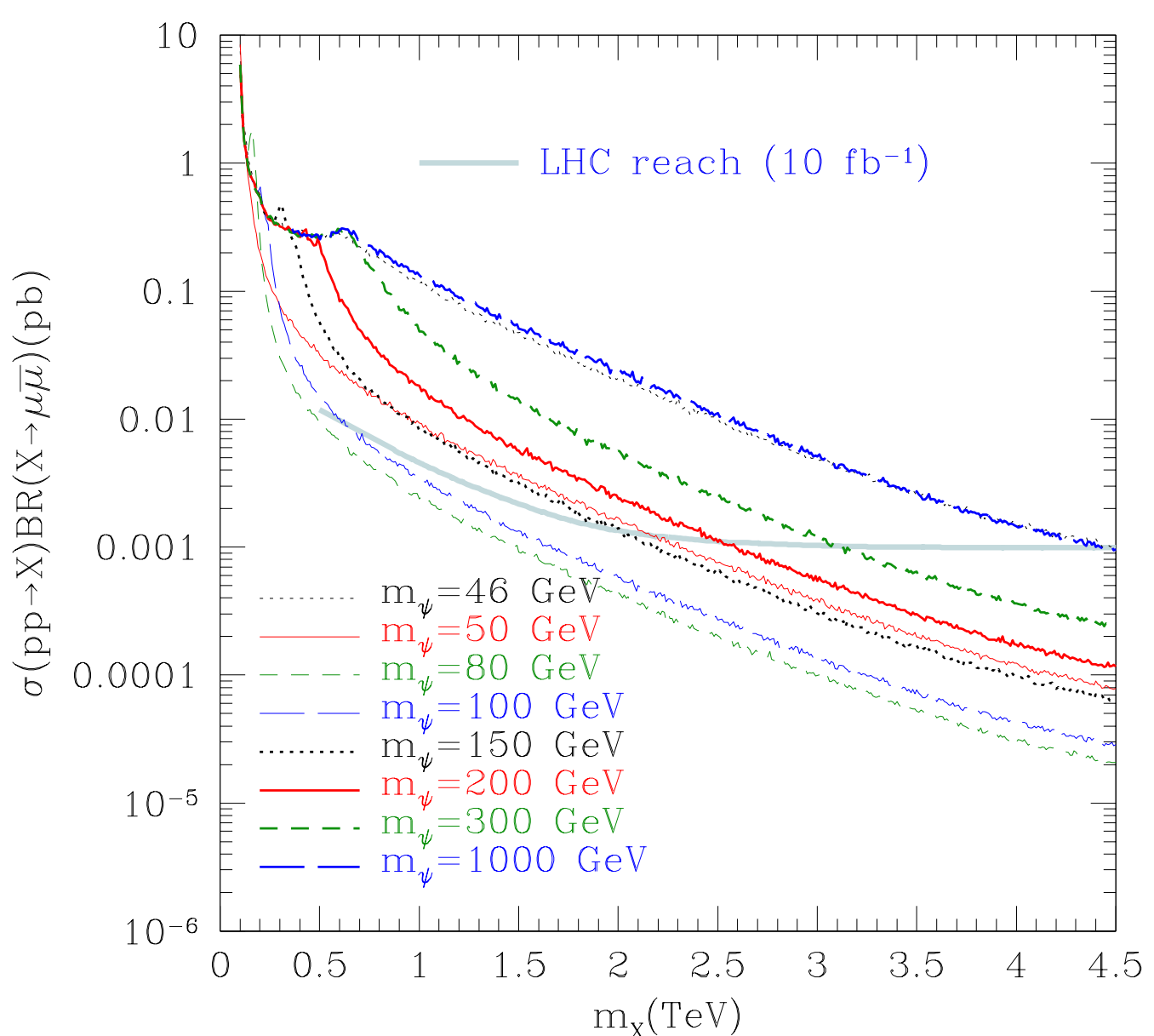

Figure 9: Maximal expected number of events for the production of the $X$ boson and its decay to $\mu \bar{\mu}$ at LHC for different values of $m_{\psi}$ compared to an estimation of the LHC sensitivity for an exposure of $10 \mathrm{fb}^{-1}$ [25]. For each value of $m_{\psi}$, the parameter $\epsilon$ is fixed to the corresponding upper bound given in Fig. 8. Notice that the curve for $m_{\psi}=1000$ $\mathrm{GeV}$ is cut at $m_{X} \sim 360 \mathrm{GeV}$ due to the perturbativity bound shown as a shaded area in Fig. 7.

In particular, we found that the current Tevatron result puts the stronger bound on the kinetic mixing term $\epsilon$ for $m_{X} \lesssim 600 \mathrm{GeV}$, while at larger masses Electro-Weak Precision Tests are more constraining. We have also found some intervals in the $m_{X}$ and $m_{\psi}$ masses $\left(m_{X} \lesssim 350 \mathrm{GeV}\right.$ and $\left.m_{\psi} \gtrsim 320 \mathrm{GeV}\right)$ that result to be excluded by a perturbativity requirement, since the combination of the relic abundance, the CDF and EWPT constraints require $\left(g_{\psi}^{X}\right)^{2} /(4 \pi)>1$. The same perturbativity constraint, combined to the requirement that the relic density of our DM candidate matches the observed value, allowed us to put also a lower bound on the $s_{\epsilon}$ parameter as a function of $m_{\chi}$ and $m_{\psi}$.

Finally, the LHC prospects for observing the $X$ gauge boson have been analyzed taking the center-of-mass energy of $14 \mathrm{TeV}$ and the integrated luminosity of $10 \mathrm{fb}^{-1}$. We found that an $X$ gauge boson mass up to $4.5 \mathrm{TeV}$ is within the LHC reach when 
the dark matter mass $m_{\psi}$ is heavy enough to suppress the invisible decay $X \rightarrow \psi \bar{\psi}$, or in the resonant limit $m_{\psi} \sim m_{Z} / 2$, when the $X \rightarrow \psi \bar{\psi}$ decay is negligible because the $X$-dark matter coupling $g_{X}$ is suppressed due to the cosmological bound on $\Omega_{\psi} h^{2}$. Moreover, an $X$ boson mass up to $2.5 \mathrm{TeV}$ can be probed if the dark matter mass is heavier than about $200 \mathrm{GeV}$. These results are obtained assuming that the kinetic mixing parameter saturates all the constraints including the Tevatron limit, and would be weakened by choosing smaller values.

Acknowledgment: SS is supported by NRF with CQUEST grant 2005-0049049 and by by the Sogang Research Grant 2010.

\section{A. Interaction couplings}

Comparing Eq. (2.13) with Eqs. (2.19) - (2.23), one can easily find the redefined couplings expressed by the physical observables (unhatted parameters):

$$
\begin{aligned}
g_{f}^{W}= & -\frac{e}{\sqrt{2} s_{W}}\left(1-\frac{\omega}{2\left(1-t_{W}^{2}\right)}\right), \\
g_{f L}^{Z}= & -\frac{e}{c_{W} s_{W}} c_{\xi}\left\{T_{3}\left[1+\frac{\omega}{2}\right]-Q\left[s_{W}^{2}+\omega\left(\frac{2-t_{W}^{2}}{2\left(1-t_{W}^{2}\right)}\right)\right]\right\}, \\
g_{f R}^{Z}= & \frac{e}{c_{W} s_{W}} c_{\xi} Q\left[s_{W}^{2}+\omega\left(\frac{2-t_{W}^{2}}{2\left(1-t_{W}^{2}\right)}\right)\right] \\
g_{\psi}^{Z}= & -g_{X} \frac{s_{\xi}}{c_{\epsilon}}, \\
g_{f L}^{X}= & -\frac{e}{c_{W} s_{W}} c_{\xi}\left\{T_{3}\left[s_{W} t_{\epsilon}-t_{\xi}+\frac{1}{2} \omega\left(t_{\xi}+\frac{s_{W} t_{W}^{2} t_{\epsilon}}{1-t_{W}^{2}}\right)\right]\right. \\
g_{f R}^{X}= & -\frac{e}{c_{W} s_{W}} c_{\xi} Q\left[s_{W}^{2} t_{\xi}-s_{W} t_{\epsilon}+\frac{1}{2} t_{W}^{2} \omega\left(\frac{t_{\xi}-s_{W} t_{\epsilon}}{1-t_{W}^{2}}\right)\right], \\
g_{\psi}^{X}= & \left.\left.-g_{X} \frac{c_{\xi}}{c_{\epsilon}}, \quad s_{W}^{2} t_{\xi}-s_{W} t_{\epsilon}+\frac{1}{2} t_{W}^{2} \omega\left(\frac{t_{\xi}-s_{W} t_{\epsilon}}{1-t_{W}^{2}}\right)\right]\right\}, \\
& g_{X Z}^{h}=\frac{m_{Z}^{2}}{v} c_{\xi}^{2} 2\left[2 s_{W} t_{\epsilon}-t_{\xi}+\omega\left(t_{\xi}+\frac{s_{W} t_{W}^{2} t_{\epsilon}}{1-t_{W}^{2}}\right)\right] . \\
& g_{W}^{Z}=\frac{e}{t_{W}} c_{\xi}\left(1-\frac{\omega}{2\left(c_{W}^{2}-s_{W}^{2}\right)}\right) \\
& g_{W}^{X}=-\frac{m_{Z}}{t_{W}} s_{\xi}\left(1-\frac{m_{Z}^{2}}{2\left(c_{W}^{2}-s_{W}^{2}\right)} c_{\xi}^{2}(1+\omega),\right. \\
c_{\xi}^{2} & \left.+t_{\xi}^{2}+s_{W}^{2} t_{\epsilon}^{2}-\omega\left(2+t_{\xi}^{2}-\frac{s_{W}^{2} t_{W}^{2} t_{\epsilon}^{2}}{1-t_{W}^{2}}\right)\right]
\end{aligned}
$$




\section{References}

[1] For a review, see, P. Langacker, Rev. Mod. Phys. 81 (2008) 1199 [arXiv:0801.1345 [hep-ph]].

[2] B. Holdom, Phys. Lett. B 166, 196 (1986).

[3] J. H. Huh, J. E. Kim, J. C. Park and S. C. Park, Phys. Rev. D 77, 123503 (2008) [arXiv:0711.3528 [astro-ph]].

[4] E. J. Chun and J. C. Park, JCAP 0902 (2009) 026 [arXiv:0812.0308 [hep-ph]].

[5] Z. Kang, T. Li, T. Liu, C. Tong and J. M. Yang, arXiv:1008.5243 [hep-ph].

[6] S. Gopalakrishna, S. J. Lee and J. D. Wells, Phys. Lett. B 680 (2009) 88 [arXiv:0904.2007 [hep-ph]].

[7] Y. Mambrini, arXiv:1006.3318 [hep-ph].

[8] K. Cheung and T. C. Yuan, JHEP 0703, 120 (2007) [arXiv:hep-ph/0701107].

[9] D. Feldman, Z. Liu and P. Nath, Phys. Rev. D 75, 115001 (2007) [arXiv:hep-ph/0702123].

[10] F. Fucito, A. Lionetto, A. Mammarella and A. Racioppi, Eur. Phys. J. C 69, 455 (2010) [arXiv:0811.1953 [hep-ph]].

[11] C. Bouchiat and P. Fayet, Phys. Lett. B 608, 87 (2005) [arXiv:hep-ph/0410260].

[12] P. Fayet, Phys. Rev. D 75, 115017 (2007) [arXiv:hep-ph/0702176].

[13] J. Kumar and J. D. Wells, Phys. Rev. D 74 (2006) 115017 [arXiv:hep-ph/0606183].

[14] W. F. Chang, J. N. Ng and J. M. S. Wu, Phys. Rev. D 74 (2006) 095005 [Erratum-ibid. D 79 (2009) 039902] [arXiv:hep-ph/0608068].

[15] Z. Ahmed et al. [The CDMS-II Collaboration], arXiv:0912.3592 [astro-ph.CO].

[16] E. Aprile et al. [XENON100 Collaboration], arXiv:1005.0380 [astro-ph.CO].

[17] K. S. Babu, C. F. Kolda and J. March-Russell, Phys. Rev. D 57, 6788 (1998) [arXiv:hep-ph/9710441].

[18] C. Amsler et al. [Particle Data Group], Phys. Lett. B 667, 1 (2008).

[19] T. Teubner, K. Hagiwara, R. Liao, A. D. Martin and D. Nomura, arXiv:1001.5401 [hep-ph].

[20] G. Bertone, D. Hooper and J. Silk, Phys. Rept. 405, 279 (2005) [arXiv:hep-ph/0404175].

[21] E. Komatsu et al., arXiv:1001.4538 [astro-ph.CO]. 
[22] G. Jungman, M. Kamionkowski and K. Griest, Phys. Rept. 267, 195 (1996) [arXiv:hep-ph/9506380].

[23] http://home.thep.lu.se/ torbjorn/Pythia.html

[24] T. Aaltonen et al. [CDF Collaboration], Phys. Rev. Lett. 102, 091805 (2009) [arXiv:0811.0053 [hep-ex]]; C. Ciobanu et al., FERMILAB-FN-0773-E (2008).

[25] I. Golutvin, P. Moissenz, V. Palichik, M. Savina and S. Shmatov, Czech. J. Phys. 54, A261 (2004) [arXiv:hep-ph/0310336]. 